\title{
POSTNATAL EXTRA-EMBRYONIC TISSUES AS A SOURCE OF MULTIPLE CELL TYPES FOR REGENERATIVE MEDICINE APPLICATIONS
}

\author{
O.S. Gubar 1, 2, *, A.E. Rodnichenko ${ }^{2,3}$, R.G. Vasyliev ${ }^{2,3}$, A.V. Zlatska ${ }^{2,3}$, D.O. Zubov ${ }^{2,3}$ \\ ${ }^{1}$ Department of Functional Genomics, Institute of Molecular Biology and Genetics, NAS of Ukraine, \\ Kyiv 03143, Ukraine \\ ${ }^{2}$ Biotechnology Laboratory ilaya.regeneration, Medical Company ilaya ${ }^{\circledR}$, Kyiv 03115, Ukraine \\ ${ }^{3}$ State Institute of Genetic and Regenerative Medicine, NAMN of Ukraine, Kyiv 04114, Ukraine
}

Aim: We aimed to isolate and characterize the cell types which could be obtained from postnatal extra-embryonic tissues. Materials and Methods: Fresh tissues (no more than $12 \mathrm{~h}$ after delivery) were used for enzymatic or explants methods of cell isolation. Obtained cultures were further maintained at 5\% oxygen. At P3 cell phenotype was assessed by fluorescence-activated cell sorting, population doubling time was calculated and the multilineage differentiation assay was performed. Results: We have isolated multiple cell types from postnatal tissues. Namely, placental mesenchymal stromal cells from placenta chorionic disc, chorionic membrane mesenchymal stromal cells (ChM-MSC) from free chorionic membrane, umbilical cord MSC (UC-MSC) from whole umbilical cord, human umbilical vein endothelial cells (HUVEC) from umbilical vein, amniotic epithelial cells (AEC) and amniotic MSC (AMSC) from amniotic membrane. All isolated cell types displayed high proliferation rate together with the typical MSC phenotype: $\mathrm{CD}^{+} \mathrm{CD}^{+} \mathrm{CD}^{+} \mathrm{CD}^{+} \mathrm{CD}^{-146}{ }^{+} \mathrm{CD} 166^{+} \mathrm{CD} 34-\mathrm{CD} 45-\mathrm{HLA}-\mathrm{DR}-$. HUVEC constitutively expressed key markers CD31 and CD309. Most MSC and AEC were capable of osteogenic and adipogenic differentiation. Conclusion: We have shown that a wide variety of cell types can be easily isolated from extra-embryonic tissues and expanded ex vivo for regenerative medicine applications. These cells possess typical MSC properties and can be considered an alternative for adult MSC obtained from bone marrow or fat, especially for allogeneic use.

Key Words: amniotic epithelial cells, amniotic mesenchymal stromal cells, chorionic membrane mesenchymal stromal cells, placental mesenchymal stromal cells, umbilical cord mesenchymal stromal cells, human umbilical vein endothelial cells.

Extra-embryonic tissues are composed of the placenta, fetal membranes, and umbilical cord. The placenta is a disc with a diameter of $15-20 \mathrm{~cm}$ and a thickness of $2-3 \mathrm{~cm}$. The fetal membranes, amnion and chorion, extend from the edges of the chorionic disc and enclose the amniotic fluid and the fetus. Placenta, amnion and umbilical cord are usually discarded after childbirth whereas they are a reserve of a variety of cell types. During last years these postnatal extraembryonic tissues have attracted much attention as a valuable source of stem cells to be used in medicine as they are readily available, and their potential use for cell therapy poses no ethical issues. Moreover, due to their early embryonic origin, extra-embryo stem cells have more plasticity comparing to adult stem cells and additionally possess immunomodulatory potential (as a part of fetomaternal tolerance mechanism), which allows their safe use even in allogeneic applications [1]. Thus we were interested in characterization and comparison of the maximal quantity of cell types

Submitted: August 4, 2017.

*Correspondence: $\quad$ E-mail: olga.gubar@gmail.com

Tel.: +38(044)2000327

Abbreviations used: AEC - amniotic epithelial cells; AMSC amniotic MSC; ChM-MSC - chorionic membrane MSC; EMT epithelial-mesenchymal transition; FACS - fluorescence-activated cell sorting; HUVEC - human umbilical vein endothelial cells; MSC - mesenchymal stromal/stem cells; PDT - population doubling time; PI-MSC - placental MSC; UC-MSC enz. - umbilical cord MSC isolated by enzymatic digestion; UC-MSC expl. - umbilical cord MSC isolated by explants method. which could be isolated from term extra-embryo tissues.

\section{MATERIALS AND METHODS}

Our experiments were done in accordance with the bioethics and biological safety norms confirmed by the Medical company ilaya ${ }^{\circledR}$ Bioethics Commission permission. All donors have signed an informed consent before tissue donation.

Cell isolation and culturing. Fresh afterbirth (less than $12 \mathrm{~h}$ after delivery) was disassembled to different tissue types and washed several times in HBSS (Sigma-Aldrich, USA) with $1 \mathrm{~g} / \mathrm{l}$ streptomycin, $1000,000 \mathrm{IU} / \mathrm{I}$ penicillin and $2.5 \mathrm{mg} / \mathrm{ml}$ amphotericin B (Sigma-Aldrich, USA). Mesenchymal stromal cells (MSC) of different origin were isolated by explants method or via enzymatic digestion. Respective tissues were minced and digested in a mix of $0.1 \%$ pronase and $0.1 \%$ collagenase type II in DMEM/F12 medium supplemented with $2 \%$ FBS (all from Sigma-Aldrich, USA) for $1 \mathrm{~h}$ at $+37^{\circ} \mathrm{C}$. The reaction mix was then diluted $5 \times$ with PBS (Sigma-Aldrich, USA) and the cells were pelleted at $800 \mathrm{~g} 10 \mathrm{~min}$ at $+4^{\circ} \mathrm{C}$. Afterwards the cells were resuspended in growth medium (a-MEM supplemented with $10 \%$ FBS, $1 \mathrm{ng} / \mathrm{ml} \mathrm{bFGF} \mathrm{(all} \mathrm{from}$ Sigma-Aldrich, USA), $200 \mu \mathrm{M}$ stable $L$-glutamine, $1 \times$ antibiotic-antimycotic solution (all from BioWest, France) and further cultured at $+37^{\circ} \mathrm{C}, 5 \% \mathrm{O}_{2}$ and $5 \%$ $\mathrm{CO}_{2}$ in a multigas incubator (Binder CB 210, Germany).

Human umbilical vein endothelial cells (HUVEC) were obtained by a brief digestion of the inner wall 
of umbilical vein by $0.1 \%$ collagenase type II in DMEM/ F12 medium (Sigma-Aldrich, USA). The cells were further cultured on collagen-coated dishes in EGM2 medium (Lonza, USA) at $+37{ }^{\circ} \mathrm{C}, 5 \% \mathrm{O}_{2}$ and $5 \%$ $\mathrm{CO}_{2}$ in a multigas incubator (Binder CB 210, Germany).

Amniotic epithelial cells (AEC) were isolated as described by Miki et al. [2]. Briefly, the amnion was separated from the chorion, extensively washed in PBS (Sigma-Aldrich, USA) until no blood clots remained. Then the tissue was briefly washed in PBS with $0.53 \mathrm{mM} \mathrm{Na}_{2}$ EDTA and digested twice in $0.05 \%$ trypsin in $0.53 \mathrm{mM} \mathrm{Na}_{2}$ EDTA (Sigma-Aldrich, USA) for 40 min at $+37^{\circ} \mathrm{C}$. Each time the reaction was stopped with double volume of a-MEM supplemented with $10 \%$ FBS (Sigma-Aldrich, USA). The cells were pelleted at $400 \mathrm{~g} 10 \mathrm{~min}$ at $+4^{\circ} \mathrm{C}$. The cell viability was assessed by Trypan Blue staining (Sigma-Aldrich, USA). Then up to $10^{5} \mathrm{cells} / \mathrm{cm}^{2}$ were seeded on collagen-coated dishes. The cells were cultured in DMEM/F12 medium supplemented with $10 \% \mathrm{FBS}, 10 \mathrm{ng} / \mathrm{ml} \mathrm{EGF}$, $1 \mathrm{ng} / \mathrm{ml} \mathrm{bFGF,} 1 \times$ sodium pyruvate, $100 \mu \mathrm{M}$ isoproterenol (all from Sigma-Aldrich, USA), $200 \mu \mathrm{M}$ stable Lglutamine, $1 \times$ antibiotic-antimycotic (all from BioWest, France) at $+37^{\circ} \mathrm{C}, 5 \% \mathrm{O}_{2}$ and $5 \% \mathrm{CO}_{2}$ in a multigas incubator (Binder CB 210, Germany).

Amniotic MSC (AMSC) were isolated by explants method from the remains of amniotic membrane trypsin digestion.

The cells were subcultured when they reached about $90 \%$ confluency. $0.05 \%$ trypsin in $0.53 \mathrm{mM} \mathrm{Na}_{2}$ EDTA (Sigma Aldrich, USA) was used for cell dissociation.

The population doubling time (PDT) was calculated using the online source: http://www.doubling-time.com/ compute.php [3].

Flow cytometry. The cell phenotype was assessed by fluorescence-activated cell sorting (FACS) on the BD FACSAria flow cytometer (BD Pharmingen, BD Horizon, USA). Staining with the monoclonal antibodies (PerCP-Cy5.5 mouse anti-human CD105, APC mouse anti-human CD73, FITC mouse anti-human CD90, PE-Cy5 mouse anti-human HLADR, PE-Cy7 mouse anti-human CD31, APC mouse anti-human CD34, FITC mouse anti-human CD45, PE-CF592 mouse anti-human CD146, BV421 mouse anti-human CD166, PF mouse anti-human CD309) was performed according to the manufacturer's instructions (BD Pharmingen, BD Horizon, USA).

Directed multilineage differentiation. Adipogenic and osteogenic differentiation were performed as described previously [4]. Briefly, adipogenic differ- entiation was performed in DMEM with high glucose content (4.5 g/l) (Sigma-Aldrich, USA) supplemented with $5 \%$ horse serum (BioWest, France), 10\% FBS, $1 \mu \mathrm{M}$ dexamethasone, $200 \mu \mathrm{M}$ indomethacine, $500 \mu \mathrm{M}$ isobutylmethylxanthine and $5 \mu \mathrm{g} / \mathrm{ml}$ insulin (all from Sigma-Aldrich, USA). After 14 days the cells were fixed and stained with Oil Red O (Sigma-Aldrich, USA).

Osteogenic differentiation was performed in DMEM with low glucose content $(1 \mathrm{~g} / \mathrm{l})$ with $10 \%$ FBS, $100 \mathrm{nM}$ dexamethasone, $10 \mathrm{mM}$ b-glycerophosphate and $50 \mu \mathrm{g} / \mathrm{ml}$ ascorbate-2-phosphate (all from SigmaAldrich, USA). After 14 or 21 days the cells were fixed and stained with BCIP/NBT Liquid Substrate System or Alizarin Red S respectively (all from Sigma-Aldrich, USA).

\section{RESULTS AND DISCUSSION}

Extra-embryonic tissues-derived cells possess MSC phenotype. Overall we obtained seven cell culture types from extra-embryonic tissues. We used enzymatic digestion to isolate cells from placenta (further referred as placental mesenchymal stromal cells - PI-MSC), from free chorionic membrane (ChM-MSC), from amniotic membrane (AEC), two cell types from umbilical cord (MSC isolated by enzymatic digestion - UC-MSC enz. and HUVEC). Two more cell types were obtained by explants method: from amniotic membrane (AMSC) and from umbilical cord (UC-MSC expl.). The cells phenotype was assessed by FACS at P3. All described cell types demonstrated high level of MSC markers expression, e.g. CD73, CD90, CD105, CD146, CD166 (except HUVEC which did not express CD90); and did not express markers of hematopoietic progenitor cells, e.g. CD34, CD45, HLA-DR (Table, Fig. 1). HUVEC additionally expressed key markers CD31 and CD309. Interestingly, AEC maintained the same level of MSC markers expression until P8 (data not shown).

$P D T$ and morphology of extra-embryonic tissues-derived cells. Along with the phenotype analysis we calculated the PDT at P1-P3 for all described cell lines. The average PDT for most cell lines was about $30 \mathrm{~h}$ (Fig. 2). However, AEC and UC-MSC enz. doubled their number in less than $24 \mathrm{~h}$, whereas the PDT for HUVEC was about $60 \mathrm{~h}$. On the whole, extra-embryonic tissues-derived cell lines demonstrated high proliferation potential with stable PDT for at least three passages.

As expected, HUVEC had epitheloid morphology, whereas all other cell lines demonstrated typical for MSC fibroblastoid morphology (Fig. 3, a-f). Interest-

Table. Flow cytometry results of surface antigen expression on extra-embryonic tissues-derived cells. Data presented as a percentage of parent population

\begin{tabular}{|c|c|c|c|c|c|c|c|c|c|c|}
\hline \multirow{2}{*}{ Cell culture } & \multicolumn{10}{|c|}{ Surface antigen expression, $\%$} \\
\hline & CD73 & CD90 & CD105 & CD146 & CD166 & CD31 & CD309 & CD34 & CD45 & HLA-DR \\
\hline AEC & 98.5 & 99.4 & 81.2 & 89.0 & 84.2 & $\mathrm{~N} / \mathrm{D}$ & $\mathrm{N} / \mathrm{D}$ & 0.3 & 0.3 & 0.9 \\
\hline AMSC & 98.1 & 99.2 & 89.6 & 94.2 & 84.5 & $\mathrm{~N} / \mathrm{D}$ & $\mathrm{N} / \mathrm{D}$ & 0.3 & 0.2 & 0.7 \\
\hline ChM-MSC & 97.7 & 98.7 & 97.6 & 98.0 & 94.0 & $\mathrm{~N} / \mathrm{D}$ & $\mathrm{N} / \mathrm{D}$ & 0.9 & 0.3 & 0.9 \\
\hline PI-MSC & 97.1 & 97.6 & 97.3 & 94.9 & 93.2 & $\mathrm{~N} / \mathrm{D}$ & $\mathrm{N} / \mathrm{D}$ & 2.2 & 1.3 & 2.7 \\
\hline UC-MSC enz. & 96.5 & 99.7 & 96.0 & 98.6 & 96.5 & $\mathrm{~N} / \mathrm{D}$ & $\mathrm{N} / \mathrm{D}$ & 0.4 & 0.4 & 1.0 \\
\hline UC-MSC expl. & 98.9 & 99.9 & 90.6 & 99.8 & 98.0 & $\mathrm{~N} / \mathrm{D}$ & $\mathrm{N} / \mathrm{D}$ & 1.4 & 0.3 & 0.6 \\
\hline HUVEC & 93.5 & 2.8 & 97.6 & 97.8 & 92.5 & 99.8 & 98.2 & 1.5 & 0.1 & 2.8 \\
\hline
\end{tabular}

Note: N/D - not determined. 


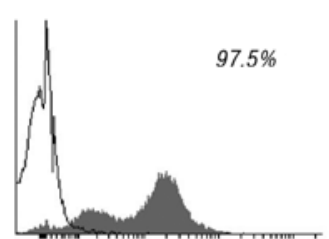

$C D 73-A P C$

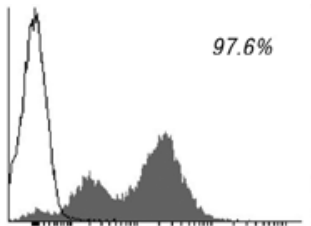

CD105 - PerCP-Cy5-5

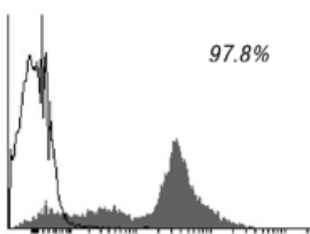

CD146 - PE-CF594

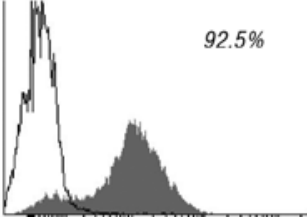

CD166 - BV421

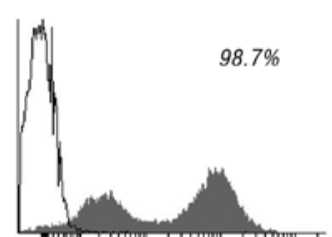

CD31-PE-Cy7

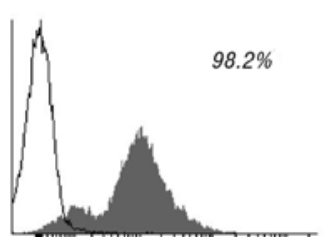

CD309 - PE

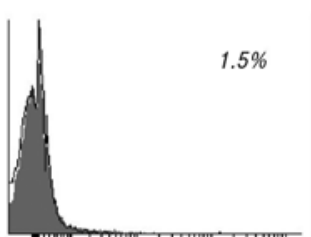

CD34 - APC

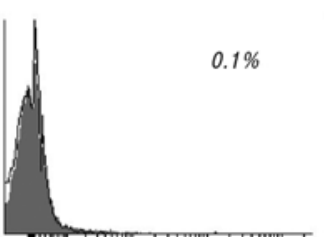

CD45 - FITC

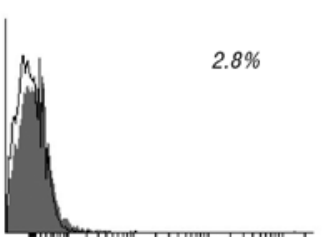

CD90 - FITC

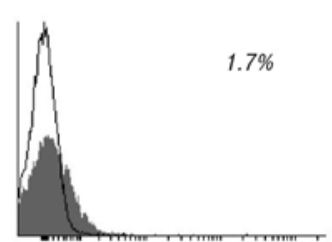

HLA-DR - PE-Cy5

Fig. 1. Representative FACS graphics (for HUVEC)

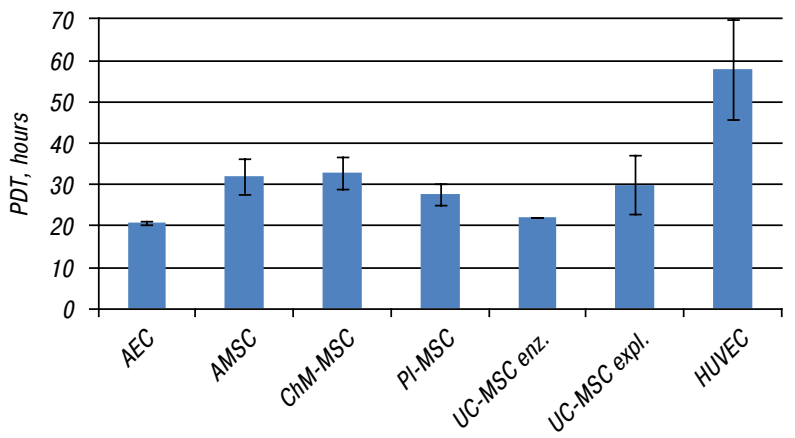

Fig. 2. PDT of extra-embryonic tissues-derived cells ingly, AEC, initially possessing typical epitheloid morphology, progressively acquired fibroblastoid appearance at P2-P3 (Fig. 3, g) probably due to the EMT [5].

Multilineage differentiation of extra-embryonic tissues-derived cells. Next, we performed adipogenic and osteogenic differentiation to assess cell plasticity of all MSC cell lines and AEC. All described cell types successfully underwent adipogenic differentiation. However, it was atypical (comparing to "classic" bone marrow MSC [6]) as there was plenty of tiny lipid vacuoles instead of several big vesicles (Fig. 4, a-f). Also AEC and most MSC cells were ca-

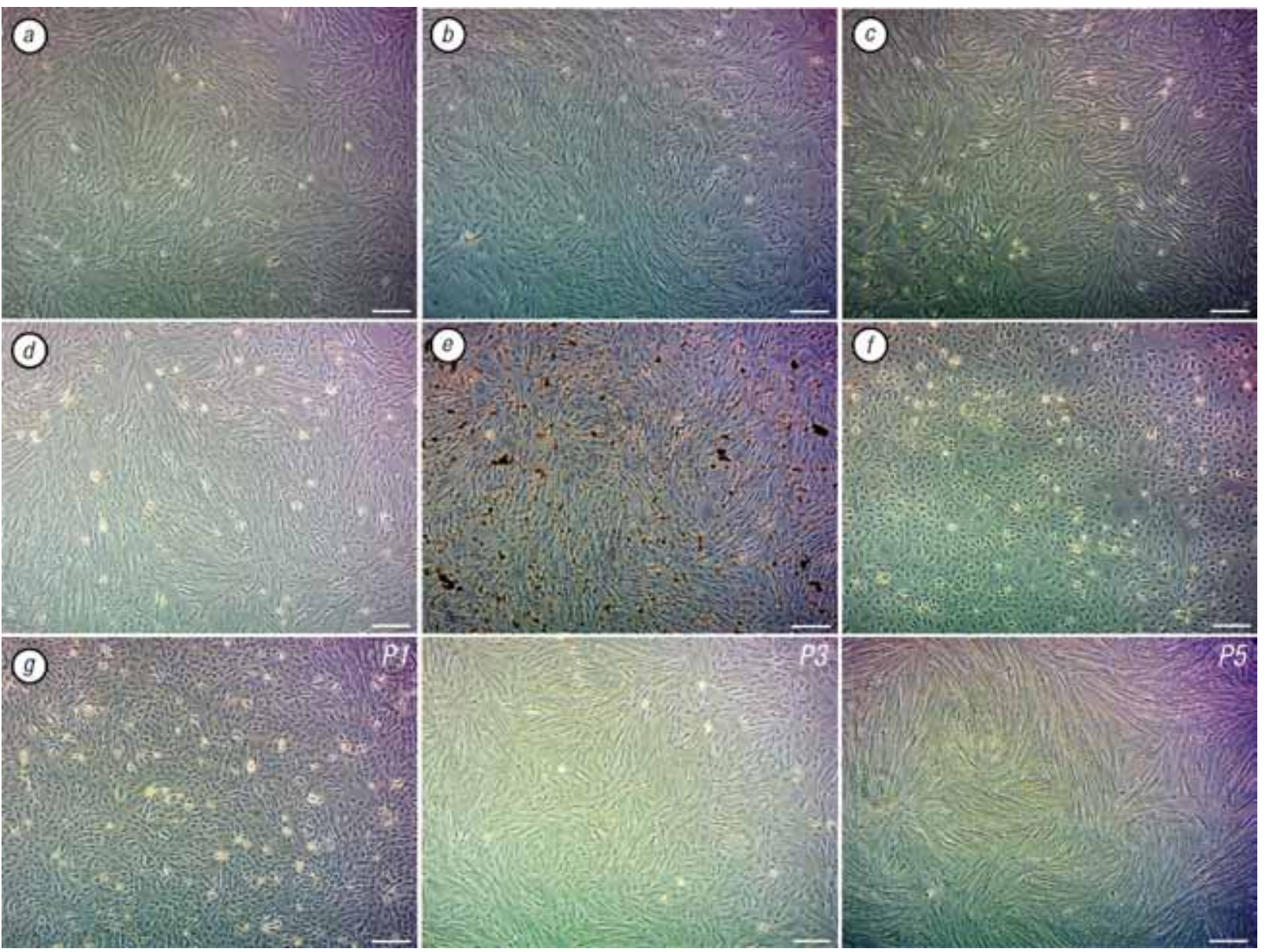

Fig. 3. Morphology of extra-embryonic tissues-derived cells. The photos are taken at $\mathrm{P} 1$. Phase-contrast microscopy. The bar $=$ $200 \mu \mathrm{m}$. $a$-AMSC; $b$-ChM-MSC; $c$-PI-MSC; $d$-UC-MSC enz.; $e$ - UC-MSC expl.; $f$-HUVEC; $g$ - epithelial-to-mesenchymal change of AEC morphology in culture. The passages are indicated in image corners 

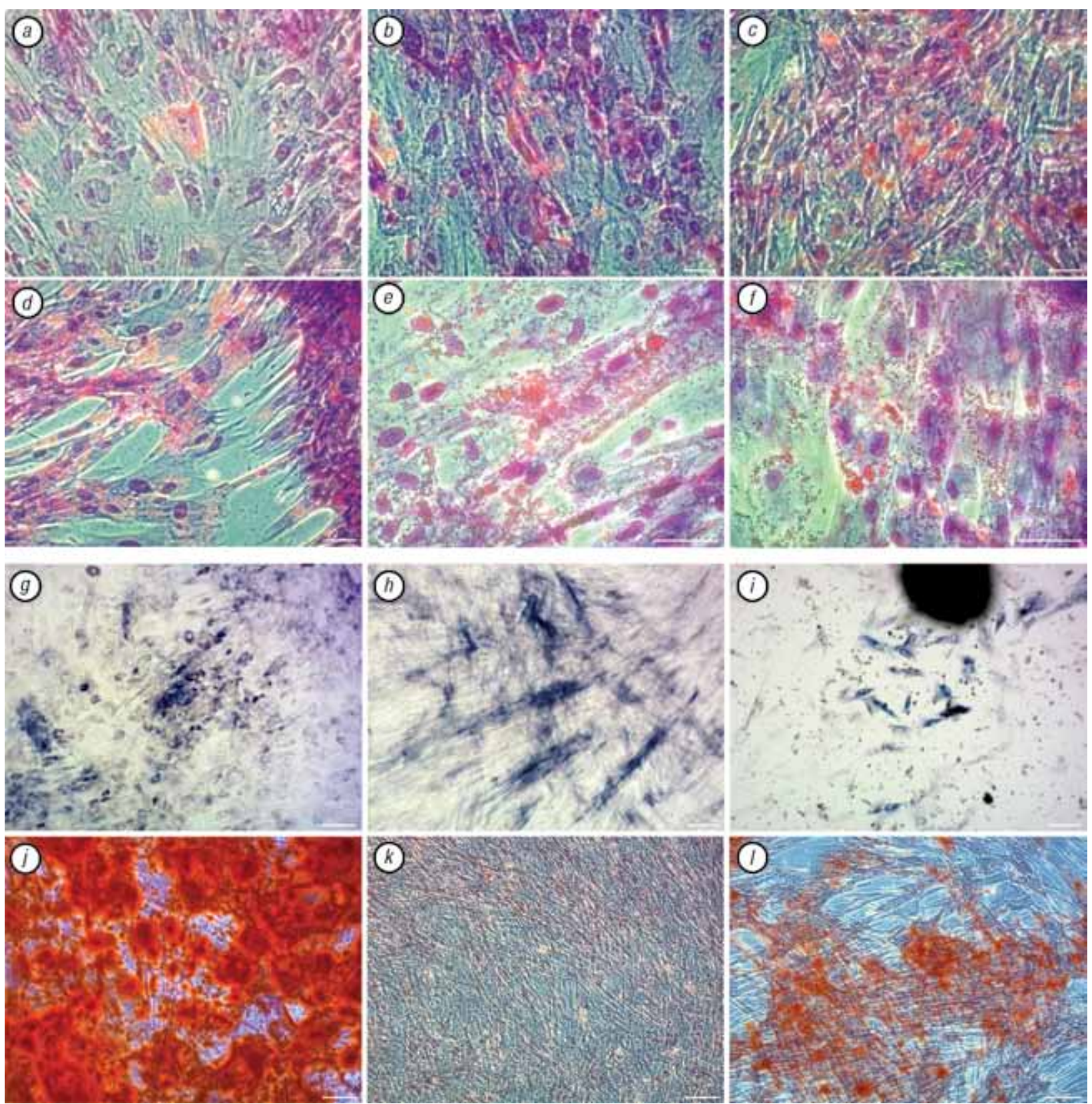

Fig. 4. Multilineage differentiation of extra-embryonic tissues-derived cells. (a-f) Adipogenic differentiation. Red Oil O and Romanowsky stains. Phase-contrast microscopy. The bar $=50 \mu \mathrm{m}$. $a-$ AEC; $b-$ AMSC; $c-$ ChM-MSC; $d-\mathrm{PI}-\mathrm{MSC} ; e-\mathrm{UC}-\mathrm{MSC}$ enz.; $f-$ UC-MSC expl. ( $g-i)$ Osteogenic differentiation. BCIP/NBT stain. Light microscopy. The bar $=100 \mu m . g-$ AEC; $h-$ ChMMSC; $i$ - PI-MSC. (j-l) Osteogenic differentiation. Alizarin Red S stain. Phase-contrast microscopy. The bar $=100 \mu \mathrm{m}$. $j-\mathrm{AMSC}$; $k$ - UC-MSC enz.; I - UC-MSC expl.

pable of osteogenic differentiation (assessed by alkaline phosphatase activity at early stages or by Alizarin Red S staining at final stages). The only exceptions were the PI-MSC and UC-MSC enz. that failed typical osteogenic differentiation. Instead PI-MSC formed spheres. Surprisingly, UC-MSC isolated via explants qualitatively demonstrated osteogenic differentiation. However, previous studies demonstrate that UC-MSC osteogenic differentiation is quite problematic and depends on multiple factors, e.g. culture conditions and even which UC segment they are isolated from [7, 8]. Probably, isolation via explants favors UC-MSC capability of mesenchymal lineage differentiation.

Thus obtained MSC cultures fulfill mostly all minimal MSC criteria [6], e.g. adherence to plastic, expression of typical MSC markers $\left(\mathrm{CD}^{2} 3^{+}, \mathrm{CD} 90^{+}, \mathrm{CD} 105^{+}\right.$and others) and multilineage differentiation. Moreover, they demonstrate high and stable proliferation rate thereby allowing obtaining a therapeutic dose in a short time. Their usage is not restricted by any ethical issues, and due to their immunophenotype (CD34-CD45-HLA-DR') they could be safely used for allogeneic applications. Two special cell types are of particular interest as they have unique properties: HUVEC and AEC. HUVEC as effective angiogenic progenitors may be used for regeneration of vessels or vascularization of complex organ transplants as well as for separate applications for correction of the tissue ischemic consequences [9]. As for AEC, they originate from embryonic epiblast, thereby capable of generating cells from all three germ 
layers (ectoderm, mesoderm and endoderm) [10]. This is of particular interest as it potentially permits liver and pancreas restoration. Moreover, several successive animal studies have already been performed in those directions [11, 12]. These outstanding features together with high accessibility make multiple cell types isolated from extra-embryonic tissues an attractive substrate for regenerative medicine applications.

\section{REFERENCES}

1. Parolini O, Alviano F, Bagnara GP, et al. Concise review: isolation and characterization of cells from human term placenta: outcome of the first international Workshop on Placenta Derived Stem Cells. Stem Cells 2008; 26: 300-11.

2. Miki T, Marongiu F, Dorko K, et al. Isolation of amniotic epithelial stem cells. Curr Protoc Stem Cell Biol 2010; 12: 1E.3.1-1E.3.10.

3. Roth V. 2006 Doubling Time Computing, Available from: http://www.doubling-time.com/compute.php.

4. Vasyliev RG, Rodnichenko AE, Zubov DA, et al. Biological properties of neural crest-derived multipotent stem cells from the bulge region of whisker follicle expanded under new culture conditions. Biopolym Cell 2014; 30: 469-76.

5. Pratama G, Vaghjiani V, Tee JY, et al. Changes in culture expanded human amniotic epithelial cells: implications for potential therapeutic applications. PLoS One 2011; 6: e26136.
6. Dominici M, Le Blanc K, Mueller I, et al. Minimal criteria for defining multipotent mesenchymal stromal cells. The International Society for Cellular Therapy position statement. Cytotherapy 2006; 8: 315-7.

7. Lee S, Park B-J, Kim J, et al. The effect of fibroblast growth factor on distinct differentiation potential of cord blood-derived unrestricted somatic stem cells and Wharton's jelly-derived mesenchymal stem/stromal cells. Cytotherapy 2015; 17: 1723-31.

8. Lim J, Razi ZRM, Law J, et al. MSCs can be differentially isolated from maternal, middle and fetal segments of the human umbilical cord. Cytotherapy 2016; 18: 1493-502.

9. Weymann A, Schmack B, Okada T, et al. Reendothelialization of human heart valve neoscaffolds using umbilical cord-derived endothelial cells. Circ J 2013; 77: 207-16.

10. Ilancheran S, Michalska A, Peh G, et al. Stem cells derived from human fetal membranes display multilineage differentiation potential. Biol Reprod 2007; 77: 577-88.

11. Marongiu M, Serra MP, Contini A, et al. Rat-derived amniotic epithelial cells differentiate into mature hepatocytes in vivo with no evidence of cell fusion. Stem Cells Dev 2015; 24: 1429-35.

12. Lin JS, Zhou L, Sagayaraj A, et al. Hepatic differentiation of human amniotic epithelial cells and in vivo therapeutic effect on animal model of cirrhosis. J Gastroenterol Hepatol 2015; 30: 1673-82. 